\title{
Nuclear Matter Effects on Jet Production at Electron-Ion Colliders
}

\author{
Hai Tao $\mathrm{Li} \oplus^{1,2,3, *}$ and Ivan Vitev ${ }^{3, \dagger}$ \\ ${ }^{1}$ HEP Division, Argonne National Laboratory, Argonne, Illinois 60439, USA \\ ${ }^{2}$ Department of Physics and Astronomy, Northwestern University, Evanston, Illinois 60208, USA \\ ${ }^{3}$ Theoretical Division, Los Alamos National Laboratory, Los Alamos, New Mexico, 87545, USA
}

(Received 2 November 2020; revised 26 March 2021; accepted 27 May 2021; published 23 June 2021)

Jet production and jet substructure in reactions with nuclei at future electron-ion colliders will play a preeminent role in the exploration of nuclear structure and the evolution of parton showers in strongly interacting matter. In the framework of soft-collinear effective theory, generalized to include in-medium interactions, we present the first theoretical study of inclusive jet cross sections and the jet charge at the electron-ion collider. Predictions for the modification of these observables in electron-gold relative to electron-proton collisions reveal how the flexible center-of-mass energies and kinematic coverage at this new facility can be used to enhance the signal and maximize the impact of the electron-nucleus program. Importantly, we demonstrate theoretically how to disentangle the effects from nuclear parton distribution functions and the ones that arise from strong final-state interactions between the jet and the nuclear medium.

DOI: 10.1103/PhysRevLett.126.252001

Introduction.-In the past decade, jets have emerged as premier diagnostics of the properties of hot nuclear matter created in heavy-ion collisions. The predicted synergy between the jet cross section suppression and jet substructure modification [1,2] has been a cornerstone of an ever growing experimental program at the Relativistic Heavy Ion Collider and the Large Hadron Collider. Examples of such recent and planned jet measurements can be found in Refs. [3-13]. At the future high-luminosity and highenergy electron-ion collider (EIC), jet production will also play a vital role-in precision tests of $\mathrm{QCD}$, the next generation studies of nucleon and nuclear structure, and cold nuclear matter tomography. Recently, various jet observables have been investigated in deep inelastic scattering (DIS), many of them with an eye on the EIC [14-24]. The overwhelming majority of those studies have focused on electron-proton $(e+p)$ reactions. In electron-ion $(e+A)$ reactions, jet observables at the EIC have been discussed in the context of small- $x$ gluon saturation physics and lepton-tagged jet acoplanarity.

To develop the $e+A$ jet physics program at the EIC, it is prudent to place emphasis on observables that have been the most illuminating and impactful in the case of heavyion collisions. Their measurement at the EIC is expected to avoid most of the heavy-ion background subtraction

Published by the American Physical Society under the terms of the Creative Commons Attribution 4.0 International license. Further distribution of this work must maintain attribution to the author(s) and the published article's title, journal citation, and DOI. Funded by SCOAP ${ }^{3}$. challenges due to the much cleaner DIS environment. Modification of jet observables in reactions with nuclei relative to the $e+p$ case may arise from initial-state effects and final-state jet-medium interactions. In this pioneering study, our goal is to maximize and isolate the latter. Recent work on light and heavy meson cross section modification at the EIC [25] has shown that it is advantageous to focus on the forward proton or nucleus going direction. The relevant EIC center-of-mass $\sqrt{s}$ energies and pseudorapidity $\eta$ correspond to moderate and large values of Bjorken $x$ - away from the gluon saturation regime. Still, initialstate effects are expected to be present as encoded by nuclear parton distribution functions (NPDFs); see Ref. [26] for a recent review. It is crucial to investigate observables that can disentangle these two distinct physics contributions.

In this Letter, we carry out the first calculation of inclusive jet production and the jet charge in electronnucleus collisions at the EIC and investigate the impact of initial- and final-state cold nuclear matter effects. The inclusive jet cross section can be expressed in a factorized form with the help of semi-inclusive jet functions (SiJFs),

$$
\begin{aligned}
E_{J} \frac{d^{3} \sigma^{l N \rightarrow J X}}{d^{3} P_{J}}= & \frac{1}{S} \sum_{i, f} \int_{0}^{1} \frac{d x}{x} \int_{0}^{1} \frac{d z}{z^{2}} f_{i / N}(x, \mu) \\
& \times \hat{\sigma}^{i \rightarrow f}(s, t, u, \mu) J_{f}\left(z, p_{T} R, \mu\right),
\end{aligned}
$$

where $f_{i / N}$ is the PDF of parton $i$ in nucleon $N . \hat{\sigma}^{i \rightarrow f}$ is the partonic cross section with initial-state parton $i$ and finalstate parton $f$, which we take up to next-to-leading order (NLO) in QCD [27]. $J_{f}$ is the SiJF initialed by parton $f$; it 
can be found in Refs. [28,29] and was derived in the framework the soft-collinear effective theory (SCET) [30-32]. When the jet radius $R$ is small, potentially large logarithms of the type $\ln R$ can be resummed by evolving the jet function from the jet scale $p_{T} R$ to the factorization scale $\mu$. Parton shower formation in nuclear matter has been described in the framework of soft-collinear effective theory with Glauber gluon interaction $\left(\mathrm{SCET}_{G}\right)[33,34]$. Hence, the SiJF formalism can be extended to heavy-ion collisions, as was first demonstrated in Refs. [35,36] for the cross sections of light and heavy flavor jet production. This universal approach has been validated by experimental measurements, most recently on the jet radius dependence of jet cross sections [37], and can be applied to $e+A$ collisions.

Different from inclusive jet cross sections, jet substructure measures the radiation pattern inside a given jet and is governed by a smaller intrinsic scale. Even though the differences between the substructure of jets in $e+p$ and $e+A$ are expected to be smaller than the differences between $p+p$ and $A+A$ collisions, we will show on the example of the jet charge [38] that nuclear effects can be identified. The average jet charge is defined as the transverse momentum $p_{T}^{i}$ weighted sum of the charges $Q_{i}$ of the jet constituents

$$
Q_{\kappa, \text { jet }}=\frac{1}{\left(p_{T}^{\mathrm{jet}}\right)^{\kappa}} \sum_{i \in \mathrm{jet}} Q_{i}\left(p_{T}^{i}\right)^{\kappa}, \quad \kappa>0,
$$

where $\kappa$ is a free parameter that must be positive for infrared safety. Its value controls the relative contribution of soft and hard particles to $Q_{\kappa, \text { jet }}$-a larger $\kappa$ would suppress direct contribution from soft particles. Studies in proton and heavy-ion collisions $[13,39,40]$ have found that the jet charge is strongly correlated with the electric charge of the parent parton and can be used to separate quark jets from antiquark jets and to pinpoint their flavor origin. A key point of this theoretical work is to demonstrate how to disentangle initial- and final-state effects for the inclusive jet cross section and the jet charge.

Cold nuclear matter effects. - In this Letter, initial-state effects are included through global-fit nuclear PDFs [41] that parametrize the inclusive DIS cross section modification in the European Muon Collaboration (EMC), antishadowing, and shadowing regions into leading-twist distributions with the assumption of collinear factorization. Isospin symmetry is implemented on account of the fact that the nucleus is a mix of protons and neutrons, $A=Z+N$, which will change the total density for up and down quarks significantly in the nuclear PDFs relative to the proton ones. Clearly, it will be important to investigate a possible way for efficient flavor tagging to test isospin symmetry in large nuclei and get a better handle on the flavor dependence of nuclear effects.
To account for final-state interactions, we make use of the medium-induced splitting kernels derived in the framework of $\mathrm{SCET}_{G}[33,42]$ and verified using a light cone wave function approach with DIS applications in mind $[43,44]$. These splitting kernels capture the medium effects on the full collinear shower dynamics. Similar to the squared matrix elements for collinear splitting, the real contribution for the medium corrections to the $i \rightarrow j k$ branching process with identified initial-state parton $i$ and final-state parton $j$ we denote as $f_{i \rightarrow j k}^{\text {med }}\left(z, \mathbf{k}_{\perp}\right)=$ $d N_{i \rightarrow j k}^{\text {med }} / d^{2} \mathbf{k}_{\perp} d z$. The virtual contributions are obtained following Ref. [45]. The collinear splitting kernels are singular when $z \rightarrow 1$, if $i=j$. It is important to note that

$$
f_{i \rightarrow j k}^{\text {tot }}\left(z, \mathbf{k}_{\perp}\right)=f_{i \rightarrow j k}^{\mathrm{vac}}\left(z, \mathbf{k}_{\perp}\right)+f_{i \rightarrow j k}^{\mathrm{med}}\left(z, \mathbf{k}_{\perp}\right),
$$

which in turn leads to a medium-induced contribution to the SiJFs and the evolution of fragmentation functions.

Because in-medium splitting kernels are calculated by integrating over the interactions in matter and the jet propagation path, they can only be obtained numerically and analytic dimensional regularization cannot be applied. The medium corrections to the SiJFs are obtained with an ultraviolet cutoff at the scale $\mu$ corresponding to the factorization scale as implemented in Refs. [35,36]. In analogy to SiJFs in vacuum, we take the relevant real and virtual contributions inside and outside of the jet cone to find

$$
\begin{aligned}
J_{q}^{\mathrm{med}}\left(z, p_{T} R, \mu\right)= & {\left[\int_{z(1-z) p_{T} R}^{\mu} d^{2} \mathbf{k}_{\perp} f_{q \rightarrow q g}^{\mathrm{med}}\left(z, \mathbf{k}_{\perp}\right)\right]_{+} } \\
& +\int_{z(1-z) p_{T} R}^{\mu} d^{2} \mathbf{k}_{\perp} f_{q \rightarrow g q}^{\mathrm{med}}\left(z, \mathbf{k}_{\perp}\right),
\end{aligned}
$$

and we use $2 E_{J} \tan R / 2 \cosh \eta \approx p_{T} R$ in Eq. (4). A similar expression can be derived for the gluon SiJF, but we note that, in the high $p_{T}$ and forward rapidity EIC kinematics that we are interested in, gluon contribution to jet production is insignificant even at NLO. In SiJFs all singularities when $z \rightarrow 1$ are regularized by the plus-distribution function that has the standard definition $\int_{0}^{1} d z g(z)[f(z)]_{+}=$ $\int_{0}^{1} d z[g(z)-g(1)] f(z)$.

Moving on to the jet charge modification at the EIC, to the order that we work the average gluon jet charge is zero due to electric charge conservation. The quark jet charge can be derived in SCET from the collinear factorization formula for measuring a hadron inside a jet [39]

$$
\begin{aligned}
\left\langle Q_{\kappa, q}\right\rangle= & \frac{\tilde{\mathcal{J}}_{q q}(E, R, \kappa, \mu)}{J_{q}(E, R, \mu)} \tilde{D}_{q}^{Q}(\kappa) \\
& \times \exp \left[\int_{1 \mathrm{GeV}}^{\mu} \frac{d \mu^{\prime}}{\mu^{\prime}} \frac{\alpha_{s}\left(\mu^{\prime}\right)}{\pi} \tilde{f}_{q \rightarrow q g}^{\mathrm{vac}(\kappa)],}\right.
\end{aligned}
$$


where $J_{q}(E, R, \mu)$ is a jet function and $\tilde{\mathcal{J}}_{q q}(E, R, \kappa, \mu)$ is the $(\kappa+1)$ th Mellin moment of the Wilson coefficient for matching the quark fragmenting jet function onto a quark fragmentation function. The perturbative NLO jet function and the matching coefficients from the jet to the hadron can be found in Refs. [46,47]. Given $\kappa$, for each jet flavor the average jet charge only depends on one nonperturbative parameter $\tilde{D}_{q}^{Q}(\kappa)$, which is obtained from PYTHIA [48] simulations, and the initial scale for the vacuum fragmentation function is set to $1 \mathrm{GeV}$. In the above equation $\tilde{f}_{q \rightarrow q g}^{\mathrm{vac}}(\kappa)$ is the $(\kappa+1)$ th Mellin moment of the splitting function $f_{q \rightarrow q g}^{\mathrm{vac}}(z)$. We found very good agreement between Eq. (5) and PYTHIA simulation for a large range of jet $p_{T}$ in both $e+p$ and $p+p$ collisions. We use the same nonperturbative parameter $\tilde{D}_{q}^{Q}(\kappa)$ for different jet radii $R$.

Nuclear matter effects on the jet charge were studied in Refs. [40,49] for the case of heavy-ion collisions. Following the derivations in Ref. [40], the average jet charge at the EIC can be written as

$$
\begin{aligned}
\left\langle Q_{\kappa, q}^{e A}\right\rangle= & \left\langle Q_{\kappa, q}^{\mathrm{ep}}\right\rangle \exp \left[\int_{\mu_{0}}^{\mu} \frac{d \mu^{\prime}}{\mu^{\prime}} \frac{\alpha_{s}\left(\mu^{\prime}\right)}{2 \pi^{2}}\left(2 \pi \mu^{\prime 2}\right) \tilde{f}_{q \rightarrow q g}^{\mathrm{med}}\left(\kappa, \mu^{\prime}\right)\right] \\
& \times\left(1+\tilde{\mathcal{J}}_{q q}^{\mathrm{med}}-J_{q}^{\mathrm{med}}\right)+\mathcal{O}\left(\alpha_{s}^{2}\right) .
\end{aligned}
$$

Here, the exponential term comes from the mediummodified Dokshitzer-Gribov-Lipatov-Altarelli-Parisi e volution from $\mu_{0} \approx \Lambda_{\mathrm{QCD}}$ to the jet scale and $\tilde{f}_{q \rightarrow q g}^{\mathrm{med}}(\kappa, \mu)=$ $\int_{0}^{1} d x\left(x^{\kappa}-1\right) f_{q \rightarrow q g}^{\operatorname{med}}(x, \mu)$. Finally, from the second line of Eq. (6), we have explicitly

$$
\begin{aligned}
\tilde{\mathcal{J}}_{q q}^{\mathrm{med}}-J_{q}^{\mathrm{med}}= & \frac{\alpha_{s}(\mu)}{\pi} \int_{0}^{1} d x\left(x^{\kappa}-1\right) \\
& \times \int_{0}^{2 E x(1-x) \tan R / 2} d^{2} \mathbf{k}_{\perp} f_{q \rightarrow q g}^{\mathrm{med}}\left(x, \mathbf{k}_{\perp}\right) .
\end{aligned}
$$

Numerical results.-In the calculations that follow, we use the CT14nlo PDF sets [50] for the proton and the nCTEQ15FullNuc PDF sets [41] for the nucleus, as provided by LHAPDF6 [51]. Consistent with Ref. [25], we fix the nominal transport coefficient of cold nuclear matter $\left\langle k_{\perp}^{2}\right\rangle / \lambda_{g}=0.12 \mathrm{GeV}^{2} / \mathrm{fm}$, consider a gold (Au) nucleus, and average over the nuclear geometry. The inmedium shower corrections induced by the interactions between the final-state parton and the nucleus vary with the parton energy in the nuclear rest frame, where the lower energy partons receive larger medium corrections. Therefore, we focus on jet production in the forward rapidity region $2<\eta<4$, where the measurement is still possible but the jet energy is lower in the nuclear rest frame. For the inclusive jet cross section, we include all partonic channels and the resolved photon contribution. Our results in $e+p$ collisions are consistent with the ones from Ref. [15].
Nuclear effects on reconstructed jets in electron-nucleus collisions can be studied through the ratio

$$
R_{e A}(R)=\frac{1}{A} \frac{\int_{\eta 1}^{\eta 2} d \sigma /\left.d \eta d p_{T}\right|_{e+A}}{\int_{\eta 1}^{\eta 2} d \sigma /\left.d \eta d p_{T}\right|_{e+p}} .
$$

The jet calculations correspond to the anti- $k_{T}$ algorithm and as a first example we choose a radius parameter $R=0.5$. The uncertainties of $R_{e A}$ are calculated by varying the scale settings in the numerator and denominator simultaneously, i.e., in a correlated way, as it minimizes the variation due to the overall normalization of cross sections. In Fig. 1, bands correspond to scale uncertainties from varying the factorization scale and the jet scale by a factor of 2 independently. For jet rapidity $\eta=2$ at leading order, when the jet transverse momentum is in the range $5-25 \mathrm{GeV}$, the Bjorken $x$ varies from 0.09 to 0.43 corresponding to the so-called antishadowing and EMC regions of nuclear PDFs. As a result, there is an enhancement for small $p_{T}$ due to antishadowing and a suppression for large $p_{T}$ due to the EMC effect, which is shown by the blue band in the upper panel of Fig. 1. The green band represents the finalstate effects, which give rise to 10\%-20\% suppression when $p_{T} \sim 5 \mathrm{GeV}$. They are smaller for larger jet energy as expected, and going to backward rapidities further reduces the effect of medium-induced parton showers. The predicted full $R_{e A}(R=0.5)$ for $18 \mathrm{GeV}(e) \times 275 \mathrm{GeV}(A)$ collisions is given by the red band. To illustrate the impact of a different NPDF choice, we show in the lower panel of Fig. 1 a comparison between the $R_{e A}$ computed with the

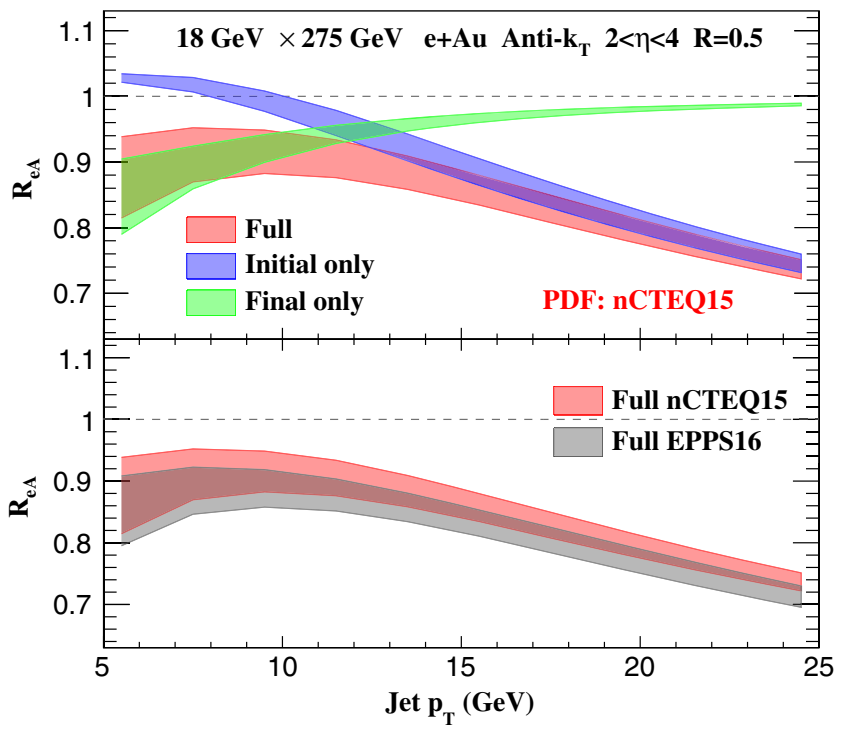

FIG. 1. Modifications of the inclusive jet cross section in $18 \mathrm{GeV} \times 275 \mathrm{GeV} e+\mathrm{Au}$ collisions for the rapidity interval $2<\eta<4$. In the upper panel, the blue and green bands represent contributions from initial-state PDFs and final-state interaction between the jet and cold nuclear matter, whereas the red band is the full result. The lower panel shows the full $R_{e A}$ for two different NPDF sets. 
nCTEQ15 [41] and EPPS16 [52] sets. We find that the difference in cross sections is less than 5\%. (Other uncertainties can arise from Monte Carlo replicas within the same PDF set or variation in the transport properties of nuclear matter.) The measurements of jet modification in the future will improve our understanding of strong interactions inside nuclei and nuclear PDFs at moderate and large Bjorken $x$.

To study cold nuclear matter transport properties with jets at the EIC, it is essential to reduce the role of NPDFs and enhance the effects due to final-state interactions. An efficient strategy is to measure the ratio of the modifications with different jet radii, $R_{e A}(R) / R_{e A}(R=1)$, as for jets with the same kinematics initial-state effects in $e+A$ reactions will cancel. This is also an observable very sensitive to the details of in-medium branching processes [1] and greatly discriminating with respect to theoretical models [37]. Furthermore, it is very beneficial to explore smaller center-of-mass energies where the final-state effects are expected to be larger, even though the cross section is smaller. Such measurements will take advantage of the high-luminosity design of the future facility. Our predictions for the ratio of jet cross section suppressions for different radii at the EIC is presented in Fig. 2, where the upper and lower panels correspond to results for $10 \mathrm{GeV}(e) \times 100 \mathrm{GeV}(A)$ and $18 \mathrm{GeV}(e) \times$ $275 \mathrm{GeV}(A)$ collisions, respectively. The plot in the upper panel is truncated around $p_{T} \sim 20 \mathrm{GeV}$ because of phase space constraints in the lower energy collisions.

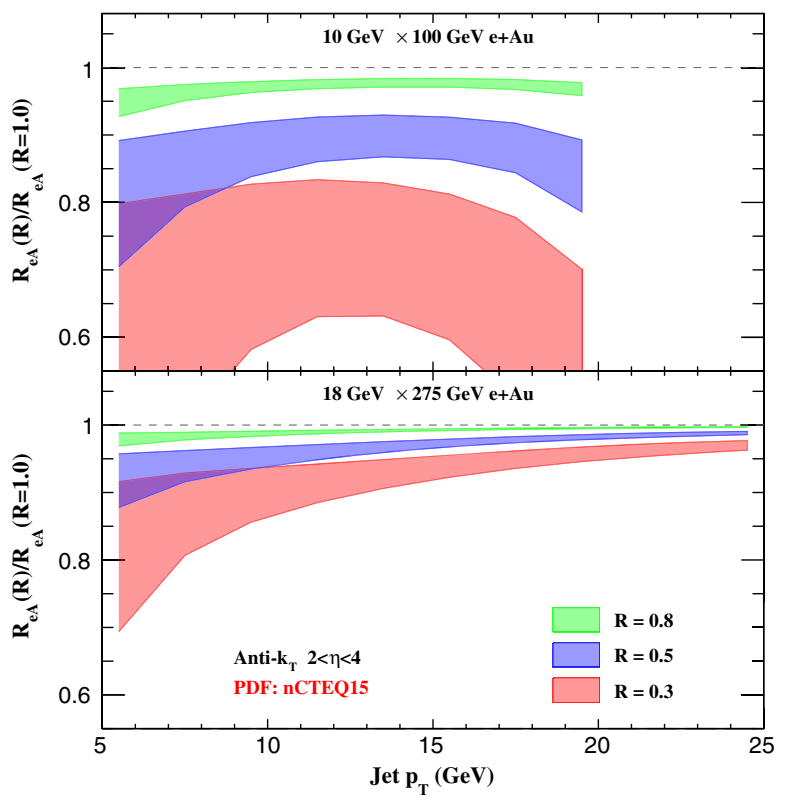

FIG. 2. Ratio of jet cross section modifications for different radii $R_{e A}(R) / R_{e A}(R=1.0)$ in $10 \mathrm{GeV} \times 100 \mathrm{GeV}$ (upper) and $18 \mathrm{GeV} \times 275 \mathrm{GeV}$ (lower) $e+$ Au collisions, where the smaller jet radius is $R=0.3,0.5$, and 0.8 , and the jet rapidity interval is $2<\eta<4$.
By comparing the $18 \mathrm{GeV} \times 275 \mathrm{GeV} e+\mathrm{Au}$ collision results to the ones in Fig. 1 , we see that $R_{e A}(R) / R_{e A}$ $(R=1)$ indeed eliminates initial-state effects. To underscore this point, in addition to using the nCTEQ15 NPDF set [41], we evaluated the double ratio with the EPPS16 [52] parametrization and found that the results are indistinguishable. The red, blue, and green bands denote ratios with $R=0.3,0.5$, and 0.8 , respectively. Since mediuminduced parton showers are broader than the ones in the vacuum, for smaller jet radii the suppression from finalstate interactions is more significant. Even though the scale uncertainties also grow, the nuclear effect is very clear and its magnitude is further significantly enhanced by the steeper $p_{T}$ spectra at lower $\sqrt{s}$.

For jet substructure, Fig. 3 presents our jet charge results at the EIC in $18 \mathrm{GeV} \times 275 \mathrm{GeV} e+$ Au collision and for radius parameter $R=0.5$. The red, blue, and green bands correspond to the jet charge parameters $\kappa=0.3,1.0$, and 2.0, see Eq. (2), respectively. The upper panel shows the modification for the average charge of up-quark initialed jets, where the rapidity is fixed to be $\eta=3$. It is defined as $\left\langle Q_{\kappa, q}^{e A}\right\rangle /\left\langle Q_{\kappa, q}^{e p}\right\rangle$ and predicted by Eq. (6), which is independent of the jet flavor and originates purely from final-state interactions. Flavor separation for jets has been accomplished at the LHC [53] and should be pursued at the EIC. For a larger $\kappa$, the $(\kappa+1)$ th Mellin moment of the splitting function is more sensitive to soft-gluon emission in that it affects the $z \sim 1$ region in the splitting function where medium enhancement for soft-gluon radiation is the largest. As shown in the upper panel of Fig. 3, the modification is

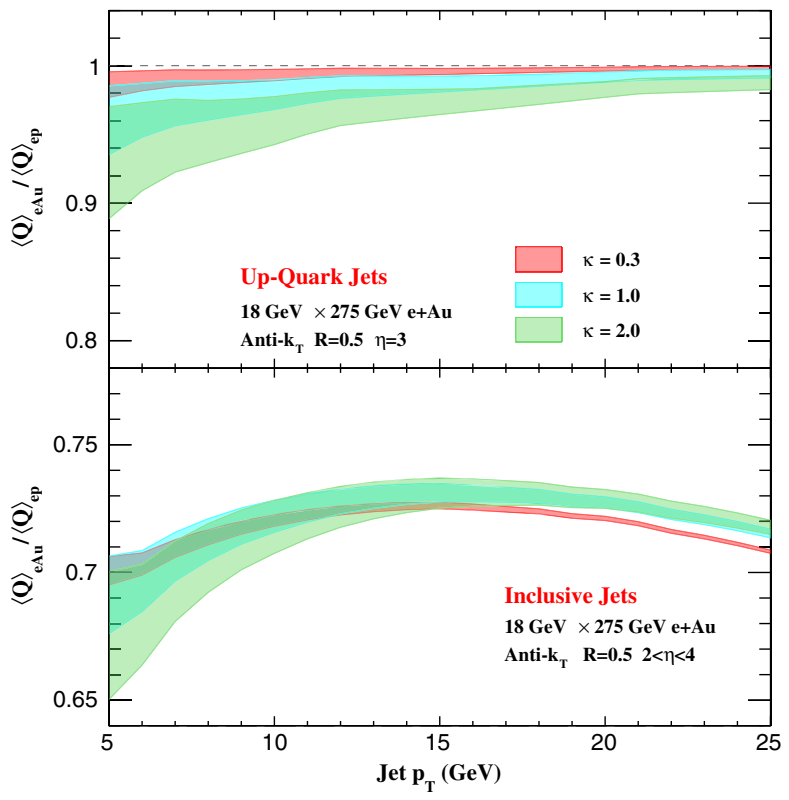

FIG. 3. Modifications of the jet charge in $e+\mathrm{Au}$ collisions. The upper panel is the modification for up-quark jet with $\eta=3$ in the lab frame. The lower panel shows results for inclusive jet with $2<\eta<4$ in $18 \mathrm{GeV} \times 275 \mathrm{GeV} e+\mathrm{Au}$ collisions. 
more significant for larger $\kappa$. The overall corrections are of order $10 \%$ or smaller and decrease with increasing $p_{T}$. The modification of the average charge for inclusive jets behaves very differently because there is a cancellation between contributions from jets initiated by different flavor partons, in particular, from up quarks and down quarks. The lower panel of Fig. 3 shows the ratio of average charges for inclusive jets with $R=0.5$ and $2<\eta<4$ for $e+A$ and $e+p$ collisions. The modification is about $30 \%$ and the $\kappa$ dependence is small due to the large difference between upand down-quark density between proton and gold PDFs. Precision measurement of the charge for inclusive jets will be an excellent way to constrain isospin effects and the upand down-quark PDFs in the nucleus.

Conclusions. - In summary, we presented a pioneering study of inclusive jet cross sections and the jet charge in electron-nucleus collisions at the EIC, building upon the SCET approach. Initial-state effects were considered via global-fit nuclear PDFs and the corrections that arise from interactions between the jet and nuclear matter were implemented with the help of the medium-induced splitting kernels derived in the framework of SCET with Glauber gluon interactions. Our results demonstrate that the forward proton-nucleus going direction is the optimal region to observe large final-state modifications due to in-medium shower evolution. We further find that final-state effects on $R_{e A}$ are large in the relatively small- $p_{T}$ region, whereas initial-state effects, if sizable, are observed at large $p_{T}$. A major advantage of jet measurements in comparison to the ones for semi-inclusive hadron production is that, by considering the ratio of cross section modifications for different jet radii, the effects from nuclear PDFs can be strongly suppressed to cleanly probe the strong interaction between jets and cold nuclear matter. With judicious choice of the center-of-mass energy, rapidity interval, and jet radius $R$, the inclusive cross section suppression can be nearly a factor of 2-similar to what is measured with high precision in $A+A$ relative to $p+p$ collisions. Related to the jet attenuation in cold nuclear matter is the modification of jet substructure. The jet charge modification of individual flavor jets can shed light on the medium-induced scaling violations in QCD; whereas a precision study of the charge of inclusive jets can be used to extract the flavor information and constrain the nuclear PDFs.

Our work is an essential step in defining the jet physics program in $e+A$ collisions at the EIC [54] and in guiding experimental focus at other proposed DIS facilities, such as the Large Hadron Electron Collider (LHeC) [55] and an electron-ion collider in China (EicC) [56]. Results from this study suggest that the center-of-mass energies of order $\mathrm{TeV}$ at the $\mathrm{LHeC}$ will eliminate medium-induced parton shower effects and the facility will be best suited to study nuclear PDFs and small- $x$ physics. Conversely, the low center-ofmass energies at the EicC are very well suited to study final-state interactions in cold nuclear matter, though the $p_{T}$ of measurements will be limited. The EIC occupies a sweet spot that ensures the broadest impact of its electron-nucleus program.

We would like to thank Z. L. Liu for useful discussions. H. T. L. and I. V. are supported by the LDRD program at LANL. I. V. is partly supported by the U.S. Department of Energy under Award No. 89233218CNA000001. H. T. L. is partly supported by the U.S. Department of Energy under Award No. DE-AC02-06CH11357 and the National Science Foundation under Grant No. NSF-1740142.

*haitao.li@northwestern.edu †ivitev@lanl.gov

[1] I. Vitev, S. Wicks, and B.-W. Zhang, J. High Energy Phys. 11 (2008) 093.

[2] I. Vitev and B.-W. Zhang, Phys. Rev. Lett. 104, 132001 (2010).

[3] A. Timilsina (PHENIX Collaboration), Nucl. Phys. A956, 637 (2016).

[4] A. Adare et al. (PHENIX Collaboration), arXiv:1501.06197.

[5] L. Adamczyk et al. (STAR Collaboration), Phys. Rev. C 96, 024905 (2017).

[6] K. Kauder (STAR Collaboration), Nucl. Part. Phys. Proc. 289-290, 137 (2017).

[7] J. Adam et al. (STAR Collaboration), Phys. Rev. C 102, 054913 (2020).

[8] S. Acharya et al. (ALICE Collaboration), J. High Energy Phys. 10 (2018) 139.

[9] S. Acharya et al. (ALICE Collaboration), Phys. Rev. C 101, 034911 (2020).

[10] M. Aaboud et al. (ATLAS Collaboration), Phys. Lett. B 790, 108 (2019).

[11] M. Aaboud et al. (ATLAS Collaboration), Phys. Rev. Lett. 123, 042001 (2019).

[12] A. M. Sirunyan et al. (CMS Collaboration), Phys. Rev. Lett. 122, 152001 (2019).

[13] A. M. Sirunyan et al. (CMS Collaboration), J. High Energy Phys. 07 (2020) 115.

[14] G. Abelof, R. Boughezal, X. Liu, and F. Petriello, Phys. Lett. B 763, 52 (2016).

[15] R. Boughezal, F. Petriello, and H. Xing, Phys. Rev. D 98, 054031 (2018).

[16] T. Gehrmann, A. Huss, J. Niehues, A. Vogt, and D. Walker, Phys. Lett. B 792, 182 (2019).

[17] S. Höche, S. Kuttimalai, and Y. Li, Phys. Rev. D 98, 114013 (2018).

[18] A. Dumitru, V. Skokov, and T. Ullrich, Phys. Rev. C 99, 015204 (2019).

[19] X. Liu, F. Ringer, W. Vogelsang, and F. Yuan, Phys. Rev. Lett. 122, 192003 (2019).

[20] E.-C. Aschenauer, K. Lee, B. S. Page, and F. Ringer, Phys. Rev. D 101, 054028 (2020).

[21] Z.-B. Kang, X. Liu, S. Mantry, and D. Y. Shao, Phys. Rev. Lett. 125, 242003 (2020). 
[22] H. T. Li, I. Vitev, and Y. J. Zhu, J. High Energy Phys. 11 (2020) 051.

[23] M. Arratia, Y. Furletova, T. Hobbs, F. Olness, and S. J. Sekula, Phys. Rev. D 103, 074023 (2021).

[24] I. Kolbé, K. Roy, F. Salazar, B. Schenke, and R. Venugopalan, J. High Energy Phys. 01 (2021) 052.

[25] H. T. Li, Z. L. Liu, and I. Vitev, Phys. Lett. B 816, 136261 (2021).

[26] J. J. Ethier and E. R. Nocera, Annu. Rev. Nucl. Part. Sci. 70, 43 (2021).

[27] P. Hinderer, M. Schlegel, and W. Vogelsang, Phys. Rev. D 92, 014001 (2015); 93, 119903(E) (2016).

[28] Z.-B. Kang, F. Ringer, and I. Vitev, J. High Energy Phys. 10 (2016) 125.

[29] L. Dai, C. Kim, and A. K. Leibovich, J. High Energy Phys. 09 (2018) 109.

[30] C. W. Bauer, S. Fleming, and M. E. Luke, Phys. Rev. D 63, 014006 (2000).

[31] C. W. Bauer, D. Pirjol, and I. W. Stewart, Phys. Rev. D 65, 054022 (2002).

[32] M. Beneke, A. P. Chapovsky, M. Diehl, and T. Feldmann, Nucl. Phys. B643, 431 (2002).

[33] G. Ovanesyan and I. Vitev, J. High Energy Phys. 06 (2011) 080.

[34] Z.-B. Kang, F. Ringer, and I. Vitev, J. High Energy Phys. 03 (2017) 146.

[35] Z.-B. Kang, F. Ringer, and I. Vitev, Phys. Lett. B 769, 242 (2017).

[36] H. T. Li and I. Vitev, J. High Energy Phys. 07 (2019) 148.

[37] CMS Collaboration, Report No. CMS-PAS-HIN-18-01, 2019.

[38] R. D. Field and R. P. Feynman, Nucl. Phys. B136, 1 (1978).

[39] D. Krohn, M. D. Schwartz, T. Lin, and W. J. Waalewijn, Phys. Rev. Lett. 110, 212001 (2013).
[40] H. T. Li and I. Vitev, Phys. Rev. D 101, 076020 (2020).

[41] K. Kovarik et al., Phys. Rev. D 93, 085037 (2016).

[42] G. Ovanesyan and I. Vitev, Phys. Lett. B 706, 371 (2012).

[43] M. D. Sievert and I. Vitev, Phys. Rev. D 98, 094010 (2018).

[44] M. D. Sievert, I. Vitev, and B. Yoon, Phys. Lett. B 795, 502 (2019).

[45] J. C. Collins and J.-W. Qiu, Phys. Rev. D 39, 1398 (1989).

[46] S. D. Ellis, C. K. Vermilion, J. R. Walsh, A. Hornig, and C. Lee, J. High Energy Phys. 11 (2010) 101.

[47] A. Jain, M. Procura, and W. J. Waalewijn, J. High Energy Phys. 05 (2011) 035.

[48] T. Sjstrand, S. Ask, J. R. Christiansen, R. Corke, N. Desai, P. Ilten, S. Mrenna, S. Prestel, C. O. Rasmussen, and P.Z. Skands, Comput. Phys. Commun. 191, 159 (2015).

[49] S.-Y. Chen, B.-W. Zhang, and E.-K. Wang, Chin. Phys. C 44, 024103 (2020).

[50] S. Dulat, T.-J. Hou, J. Gao, M. Guzzi, J. Huston, P. Nadolsky, J. Pumplin, C. Schmidt, D. Stump, and C. P. Yuan, Phys. Rev. D 93, 033006 (2016).

[51] A. Buckley, J. Ferrando, S. Lloyd, K. Nordstrm, B. Page, M. Rfenacht, M. Schnherr, and G. Watt, Eur. Phys. J. C 75, 132 (2015).

[52] K. J. Eskola, P. Paakkinen, H. Paukkunen, and C. A. Salgado, Eur. Phys. J. C 77, 163 (2017).

[53] G. Aad et al. (ATLAS Collaboration), Phys. Rev. D 93, 052003 (2016).

[54] R. A. Khalek et al., arXiv:2103.05419.

[55] M. Klein, in From My Vast Repertoire ... (World Scientific, Singapore, 2019), pp. 303-347, https://doi.org/10.1142/ 9789813238053_0015.

[56] X. Chen, Proc. Sci., DIS2018 (2018) 170 [arXiv:1809.00448]. 